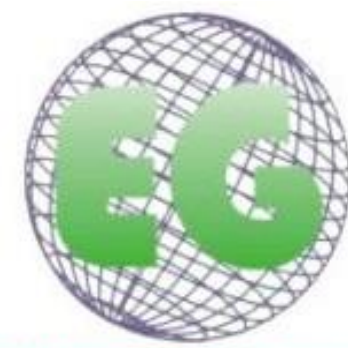

ISSN 1695-6141 $N^{\circ} 49$

\title{
Estudio de la eficacia del test TNO en la detección precoz de la ambliopía en atención primaria
}

Investigation of the Efficiency of the TNO Test for the Early Detection of Amblyopia in Primary Care

\section{Carlos Delgado Espinosa ${ }^{1}$}

Josefina G. Castañeda Suardíaz ${ }^{2}$

Noelia Cordero Jorge ${ }^{1}$

Sarai Rodríguez Rodríguez ${ }^{1}$

\author{
${ }^{1}$ Enfermero/a Servicio Canario de Salud. Tenerife. España. \\ ${ }^{2}$ Universidad de la Laguna, Sección Enfermería y Fisioterapia. Tenerife. España. \\ E-mail: carlos93lg@gmail.com
}

\section{http://dx.doi.org/10.6018/eglobal.17.1.275081}

Recibido: 15/11/2016

Aceptado: 15/04/2017

\section{RESUMEN:}

La ambliopía es la causa prevenible más común de pérdida de visión en los países desarrollados, afectando al 2-5\% de la población, se puede desarrollar debido a una experiencia visual anómala, durante la fase del crecimiento visual. El estrabismo es la principal causa del desarrollo de la ambliopía, dándose en el 30-50\% de los casos. Se considera de primordial importancia la detección temprana de las alteraciones visuales causales de la ambliopía ya que su tratamiento es sólo efectivo en la infancia, mientras se produce la maduración del sistema visual.

La Consejería de Sanidad del Gobierno de Canarias, en el ánimo de la prevención de la salud, desarrolla desde hace varios años un Programa de Detección de Alteraciones Visuales en la infancia, y recientemente ha incorporado a las exploraciones habituales la implantación del Test TNO. Esta investigación precisamente pretende valorar la eficacia del Test TNO en la detección precoz de la ambliopía y su comparación con las demás pruebas habituales realizadas en Atención Primaria.

En el estudio se realiza una exhaustiva revisión y análisis comparativo de las pruebas de visión de 358 historias clínicas de un centro de salud de la provincia de Santa Cruz de Tenerife, correspondiente a niños entre 3 y 6 años de edad.

Según los resultados obtenidos, se puede conjeturar que el test TNO es una prueba igual o más valida en la detección de anomalías que puedan servir en la detección precoz de la ambliopía, en comparación con los test más populares utilizados habitualmente en un centro de Atención Primaria para la detección de estrabismo y ambliopía.

Palabras clave: Ambliopía; estrabismo; visión binocular; Test TNO; niños, agudeza visual.

\section{ABSTRACT:}

Ambliopia is the most common preventable cause of the loss of vision in developed countries, affecting the $2-5 \%$ of the population. This may evolve due to an abnormal visual experience, during the phase of visual development. Strabismus is the principal cause of the development of ambliopia, being $30-50 \%$ 
of the cases. The early detection of visual alterations causing ambliopia is considered to be of primary importance as the treatment is only effective during infancy, when the visual system becomes mature.

The Health Council of the Canary Island Government a few years ago developed a program for the detection of visual alterations in childhood, and has recently incorporated the introduction of the TNO test to the habitual examinations. This investigation precisely intends to value the efficiency of the TNO Test in the early detection of ambliopia and the comparison with other habitual tests that are carried out in Primary Care.

During the study, an exhaustive revision and comparative analysis is made of the vision tests of 358 medical records in a health centre in the province of Santa Cruz de Tenerife, corresponding to children between 3 and 6 years old.

According to the results obtained, we can surmise that the TNO test is a valid test for the detection of anomalies, which can be used in the early detection of ambliopia, in comparison to the most popular tests used habitually in a health care centre for the detection of strabismus and ambliopia.

Keywords: Amblyopia; strabismus; binocular vision; Test TNO; children; visual acuity.

\section{INTRODUCCIÓN}

La visión es la capacidad de percibir el entorno que rodea al individuo a través de la interpretación de los rayos de luz que inciden sobre él, más concretamente sobre los ojos. Es la habilidad sensorial más relacionada con la comunicación y la relación social. Por lo tanto, la ausencia o disminución de la función visual supone una minusvalía grave, que puede dar lugar a importantes dificultades en la capacidad de aprendizaje y en la relación social de las personas.

Entre los parámetros medibles de la visión cabe destacar la visión binocular que, funcionalmente bien desarrollada, permite la estereopsis o percepción binocular de profundidad, esto es, la visión tridimensional o visión estereoscópica. El sistema visual alcanza de forma gradual una maduración casi completa en los primeros tres años de vida, aunque persiste cierta plasticidad entre los 3 y los 6 años. ${ }^{(1)}$

Como principales alteraciones de la visión cabe destacar el estrabismo, la ambliopía, los defectos de refracción, las discromatopsias y las alteraciones de la fusión. Ambliopía u "ojo vago" se define como la reducción unilateral (en la mayoría de los casos) de la agudeza visual, causada por una mala estimulación visual del cerebro durante el período crítico del desarrollo cerebral de las funciones visuales. La ambliopía se desarrolla debido a una experiencia visual anormal en fases tempranas de la vida, es la causa prevenible más común de pérdida de visión en los países desarrollados, dándose cifras del $2-5 \%$ de la población general e incluso de más de un $5 \%$ en preescolares. ${ }^{(2)}$

Dentro de los tipos de ambliopía, la estrábica es aquella que se presenta con mayor frecuencia, de forma que existe un ojo desviado que tiene un cierto grado de deprivación de su imagen enfocada, debido al mecanismo de supresión del cerebro que recibe imágenes fuera de foco que desecha. En este sentido, el estrabismo es la desalineación ocular o desviación del eje visual, ocasionada bien por anomalías de la motilidad ocular o en la visión binocular; el estrabismo afecta al 4-6\% de la población general y de ellos el 30-50\% desarrollarán ambliopía. (2) En la niñez, uno de los principales factores de riesgo para la aparición de estrabismo son las anomalías en el desarrollo cerebral, significándose que en recién nacidos sanos la incidencia de estrabismo se estima en $0,5-1 \%$. ${ }^{(3)}$

Una vez que se ha completado la maduración visual, alrededor de los 6-8 años, desaparece el riesgo de aparición de ambliopía; asimismo, su tratamiento/corrección será menos efectivo o incluso ineficaz si se inicia tardíamente, una vez completado el 
desarrollo visual. El éxito del tratamiento depende de una detección y de un tratamiento precoz durante la infancia. ${ }^{(1-5)}$ Se considera de primordial importancia la detección temprana de las alteraciones visuales causales de la ambliopía, ya que su tratamiento es sólo efectivo durante la maduración del sistema visual en la infancia, evitando de esta forma las negativas consecuencias de una visión inadecuada en la etapa adulta.

La detección precoz de trastornos visuales implica la utilización de métodos diagnósticos como el Test de Hirschberg, Cover test, agudeza visual con Optotipos, y test de visión estereoscópica. Entre los test de visión estereoscópica más conocidos se encuentra el Test TNO cuya primera edición fue diseñada en 1972 por el Departamento de percepción de la compañía The Netherlands Organisation (TNO) basada en la investigación de la ciencia aplicada, ${ }^{(6)}$ su utilización en Atención Primaria no supone un gran gasto extra de material.

Hoy en día en España, cada comunidad autónoma se encarga de gestionar sus propios recursos y programas de salud, por ello existe una serie de protocolos de actuación específicos de cada región, que los centros sanitarios deben llevar a cabo para controlar el desarrollo en la infancia, lo que implica un control de la evolución visual. Esta gestión descentralizada conlleva que existan diferencias en la gestión y desarrollo de los diferentes programas y protocolos de salud.

La Consejería de Sanidad del Gobierno de Canarias, en el ámbito de sus competencias y en el ánimo de la prevención de la salud, desarrolla desde hace varios años un Programa de Detección de Alteraciones Visuales en la infancia, y recientemente, desde el año 2012, ha incorporado a las exploraciones habituales la implantación del test TNO o de visión estereoscópica.

El objetivo principal de este estudio es determinar la utilidad del test TNO en la determinación de alteraciones visuales en la población infantil menor de seis años en un Centro de Atención Primaria.

Como objetivos específicos se ha planteado:

- Valorar y comparar los test visuales más utilizados en revisiones periódicas infantiles en un Centro de Atención Primaria.

- Determinar qué tests visuales resultan más efectivos en la prevención y detección de posibles patologías visuales durante la infancia.

- Determinar la incidencia de alteraciones visuales detectadas en un Centro de Atención Primaria.

- Comprobar y comparar la incidencia y naturaleza de las alteraciones visuales patológicas según resultado del estudio oftalmológico completo.

\section{MATERIAL Y MÉTODOS}

Este estudio pretende valorar la eficacia del Test TNO en la detección precoz de la ambliopía en un centro de Atención Primaria de la provincia de Santa Cruz de Tenerife. Se realiza una exhaustiva revisión de las historias clínicas del periodo comprendido entre el año 2012 y 2014. 
La población objeto de estudio se corresponde con la población infantil entre tres y seis años. Se trata precisamente de una edad en la que las vías visuales se encuentran en uno de los periodos más críticos de crecimiento, en el que sufrir una alteración condicionaría el correcto desarrollo visual de la infancia.

Dentro de las historias de los pacientes se valora y analiza el resultado de las pruebas y apartados de visión ubicados en la anamnesis estructurada en la sección de valoración, concretamente en la unidad de exploración/pruebas, correspondientes a las revisiones infantiles que se hayan realizado. Se trata esencialmente del Test de Hirschberg, Cover Test, agudeza visual con Optotipos y Test TNO.

\section{RESULTADOS}

La población a estudio, 358 niños, se obtuvo de la recogida de datos de tres consultas diferentes, aportando cada una un porcentaje similar de individuos. Concretamente se trató de 182 niños (51\%) y 176 niñas (49\%). Para la toma de datos y su estudio posterior se clasificó por edades, en grupos de tres, cuatro, cinco y seis años, con una media de edad de 4,84 años.

Sólo al 36\% (132 niños) se les pudo realizar todas las pruebas durante sus revisiones de salud, lo que sucede por varios motivos: la no asistencia a las revisiones, la falta de colaboración, la no realización de la prueba o la falta de constancia de ello en las historias; en el $48 \%$ de los casos (169 niños) el estudio estaba incompleto, y en el $16 \%$ de los casos (57 niños) no se ha registrado ninguna exploración. En la figura 1 se distribuye la realización de pruebas según edades.

Figura 1. Diagrama por edades de realización de pruebas

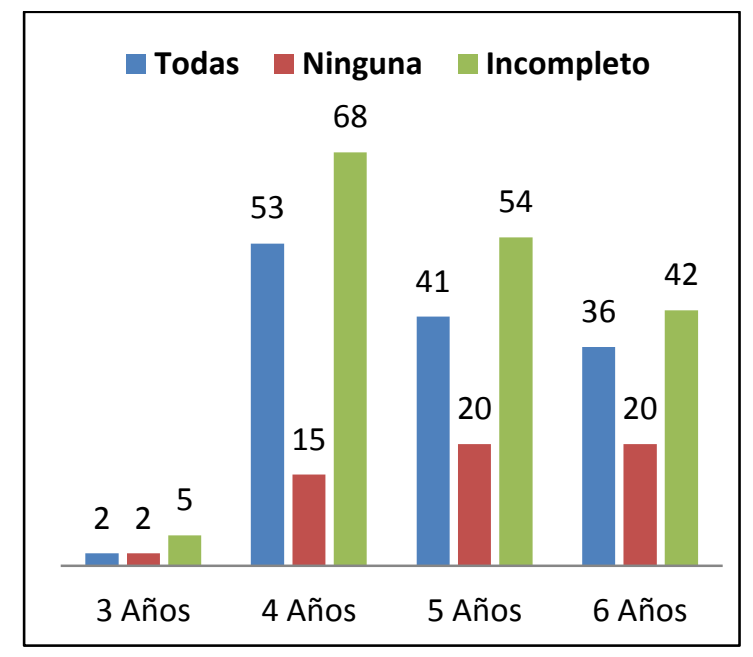

En las revisiones se examina en primer lugar si existe una aparente desviación del eje visual, cuya manifestación sea habitualmente espontánea (lo que indicaría un estrabismo). En total entre las tres consultas estudiadas hubo constancia de la existencia o no de esta desviación ocular a 256 niños, de los cuales a 8 se les detectó una posible desviación del eje visual (tropia); es decir, en 248 casos no se detectó inicialmente esta patología y en 102 no constó el registro. La figura 2 revela la distribución por edades en cuanto a la determinación de estrabismo. 
Figura 2. Diagrama según estrabismo

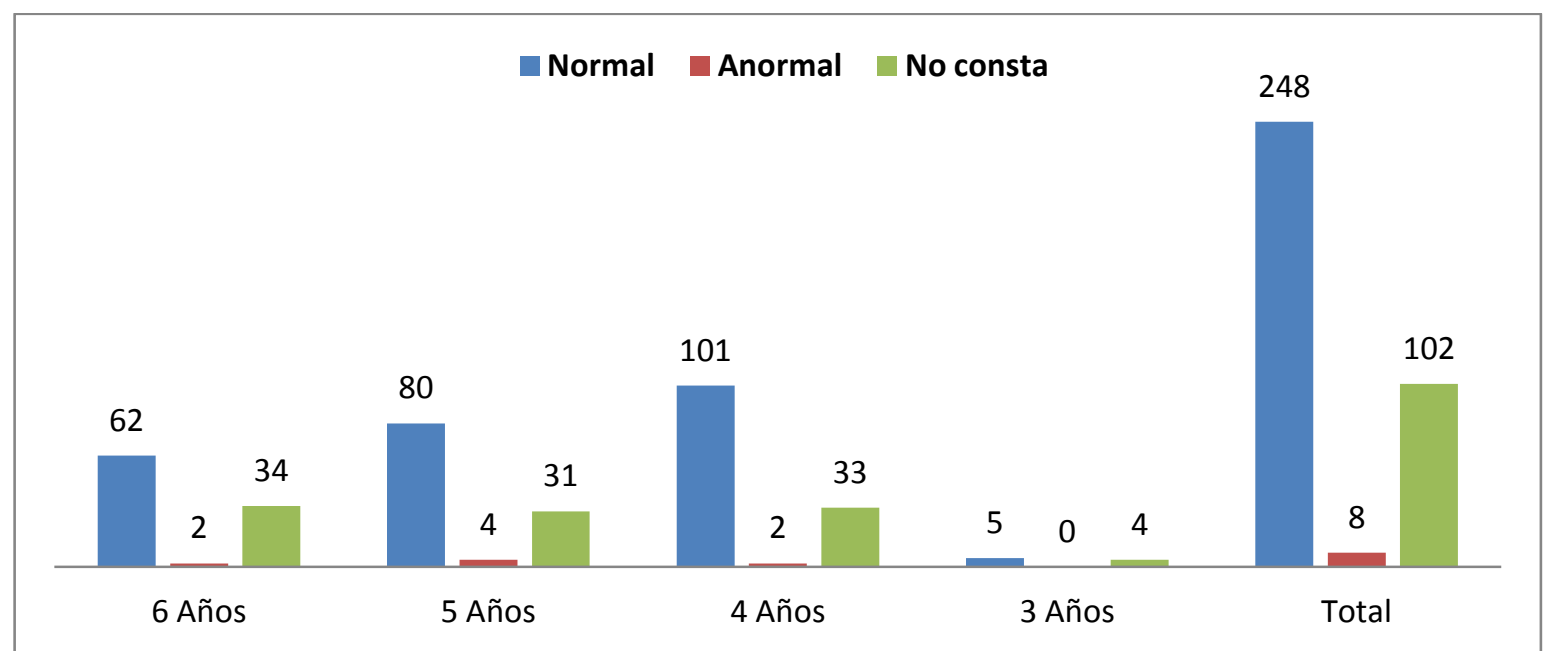

El test de Hirschberg, junto al Cover Test, es uno de los test más conocidos y usados en la detección de posibles forias en los ojos, que pudieran indicar un posible estrabismo. En total se efectuó el Test de Hirschberg a 217 niños, ya que de los 358 casos no constaba la realización en 141; de los casos revisados sólo hay constancia que se presentara anomalía en uno de ellos (figura 3). El caso específico con anomalía en la realización del test, también presentaba un estrabismo aparente según la historia de salud. En comparación con las demás pruebas a estudio, no se pudo obtener ninguna relación, ya que no había constancia de su realización en la historia del paciente (Cover Test, Agudeza visual con Optotipos o TNO). Sí se reflejaba una derivación para su seguimiento por Oftalmología, pero no existía ninguna respuesta o correspondencia en la historia de salud que este seguimiento se estuviera o se hubiera llevado a cabo.

Figura 3. Test de Hirschberg

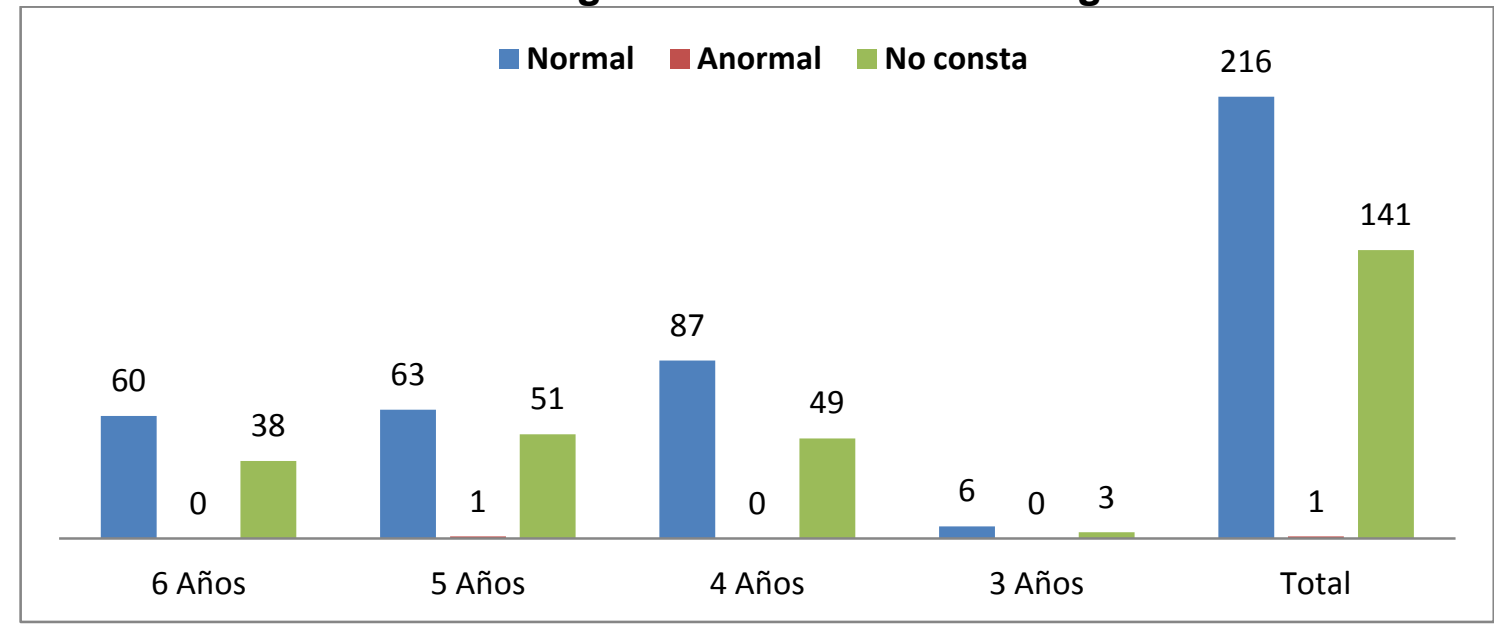

El Cover Test se realizó a un total de 168 niños, sólo hubo constancia de que dos de ellos presentaban una anomalía durante su realización. La figura 4 representa el resultado del test por edades. Uno de los casos anómalos también presentaba un estrabismo aparente en la consulta, manifestado previamente por los padres; en comparación con las demás pruebas a estudio, también se detectó una posible anomalía en la agudeza visual a través de la medición con Optotipos, no constando resultados del Test de Hirschberg y del TNO. En dicho caso existe constancia de una 
derivación para su seguimiento por Oftalmología y en la historia clínica aparece que, según refieren los padres, se le diagnosticó de hipermetropía y astigmatismo, motivo por el cual usaba lentes correctoras. El segundo caso presentaba un estrabismo aparente en la consulta, además de haber dado también un resultado anómalo tanto en la realización del TNO, como en la agudeza visual con Optotipos, siendo normal el test de Hirschberg; no existían más datos oftalmológicos del paciente ya que se refería que estaba en seguimiento por un oftalmólogo privado.

Figura 4. Cover Test

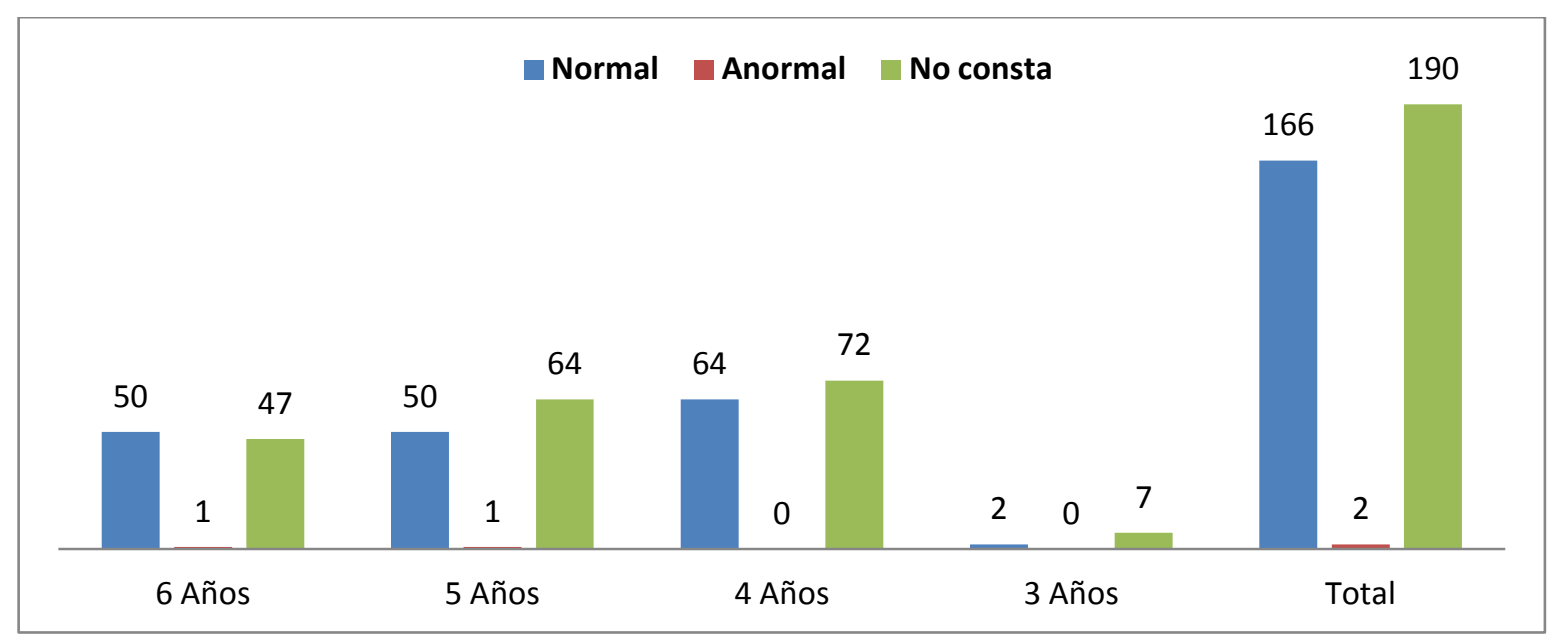

La medición de la agudeza visual a través de Optotipos es una prueba fundamental para saber si un sujeto tiene alguna complicación o problema que le impida ver correctamente por alguno de sus ojos; por ello, esta prueba se encuentra señalada como exploración mínima dentro de las valoraciones de los pacientes. Por otro lado, la medición de la agudeza visual con Optotipos, aun siendo la prueba que permite detectar más problemas de visión, también es una de las pruebas que exige mayor colaboración por parte del paciente, por lo que muchas veces no es posible su realización debido a la poca edad de los niños.

La medición de la agudeza visual con Optotipos se efectúo a 249 niños, 12 de ellos tuvieron alguna complicación o anomalía durante la prueba; 9 de ellos fueron derivados a Oftalmología para su estudio, y en los otros 3 casos restantes, al no tener grandes complicaciones, se les asignó una nueva cita dentro de varios meses para nueva valoración. La figura 5 expone la valoración de este tipo de prueba visual según edad. 
Figura 5. Agudeza visual con Optotipos

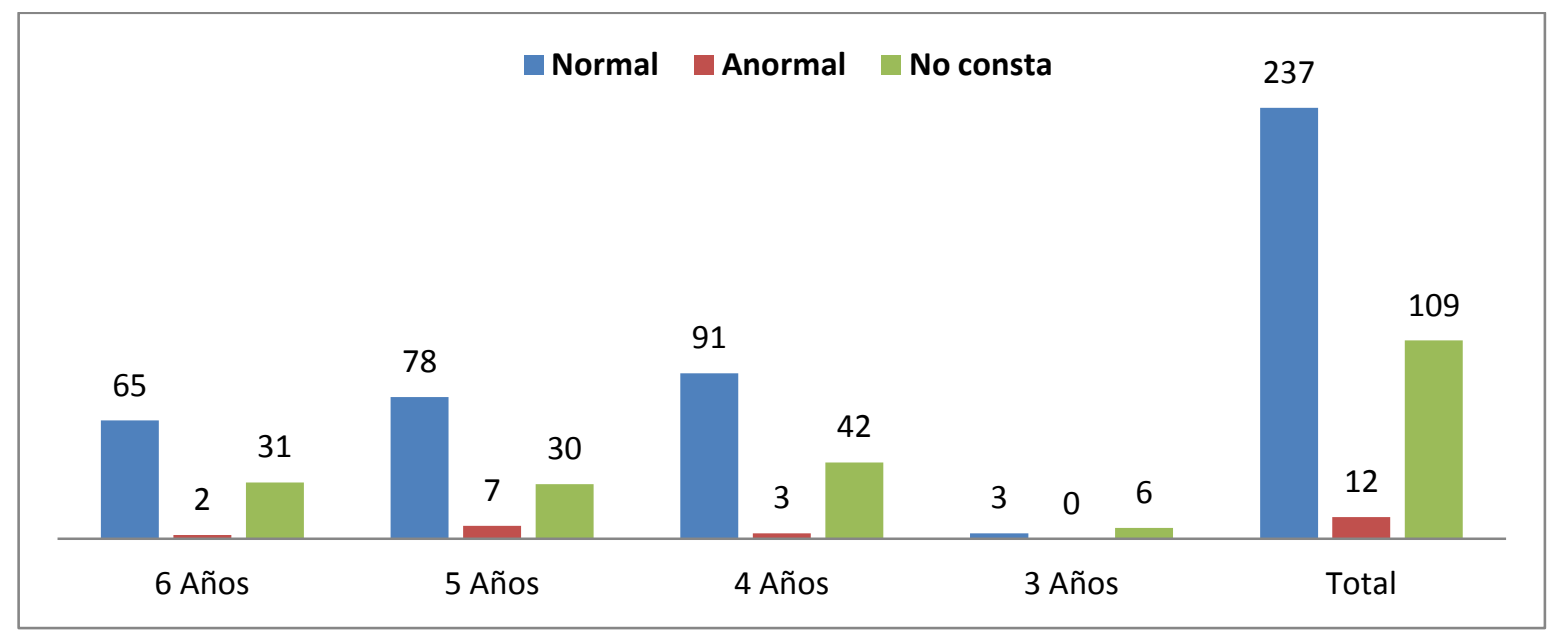

Ante el gran porcentaje de la no constancia de información y realización de las pruebas a estudio, se ha querido comparar los diferentes grados de ello, en las distintas pruebas, tal y como se muestra en la figura 6 . En el análisis de los resultados se puede comprobar cómo la medición de la agudeza visual con Optotipos es la prueba más utilizada $(70 \%)$, a pesar de ser una de las que necesita una mayor colaboración por parte del paciente.

Figura 6. Comparativa porcentaje realización de pruebas

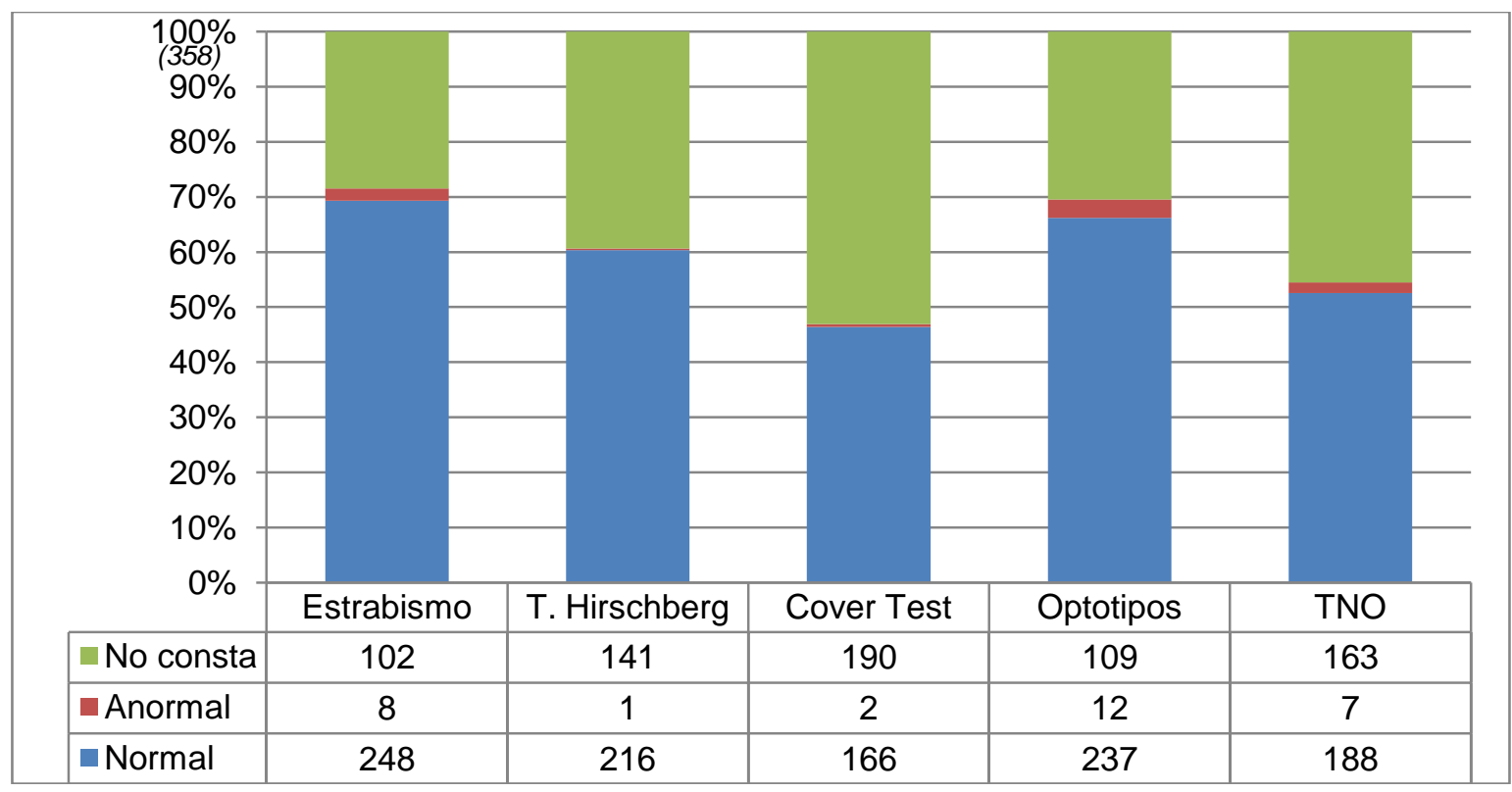

En la comparación de las pruebas del estudio, aunque no se cubrieron las expectativas en cuanto a la comprobación de la realización de las mismas, sí se analizaron suficientes casos como para poder detectar al menos un caso negativo o anormal en cada una de ellas; el mayor número de casos anormales se aprecia en las pruebas de Optotipos (12 casos), Estrabismo (8 casos) y TNO (7 casos), detectándose solamente 2 casos en el Cover Test y un caso en el Test de Hirschberg. 


\section{DISCUSIÓN}

En este estudio la prueba de agudeza visual con Optotipos es la más utilizada. Se puede suponer que esto ocurre en mayor medida a que es el único apartado/prueba, dentro de la exploración de la visión, que se encuentra remarcado en las historias clínicas como prueba obligatoria. También se cree que, al igual que en otros estudios $^{(7)}$ la falta de materiales en todas las consultas ha podido influir en la realización y resultados de las pruebas. En relación con otras pruebas del estudio, los resultados del test TNO no reflejan una dificultad en la realización del test, aun siendo una de las pruebas en la que es necesario un mayor grado de colaboración por parte del paciente.

Resalta en cuanto a hallazgos en la detección de posibles anomalías, el examen de la medición de agudeza visual con Optotipos. Este resultado puede estar directamente relacionado con la alta constancia de realización de la prueba en comparación con los demás test del estudio. También cabe destacar los bajos resultados del T. Hirschberg y el Cover Test, a pesar de ser estos unos de los test más populares y reconocidos.

Según los resultados obtenidos, se puede deducir que el test TNO es una prueba igual o más valida en la detección de anomalías que puedan servir para la detección precoz de la ambliopía, como los test específicos más populares (T. Hirschberg y Cover Test) para la detección de estrabismo y ambliopía.

En el estudio no se pudo comprobar y comparar la incidencia de las alteraciones visuales patológicas según el resultado de un estudio oftalmológico completo, ya que en las historias clínicas de los casos derivados para su valoración, no se encontró información remitida por el servicio de Oftalmología. En consecuencia, no existe una comunicación bilateral adecuada entre Atención Primaria y sus derivaciones a la interconsulta de Oftalmología. La falta de notificación entre las diferentes consultas es también la principal razón por la que no se puede especificar correctamente la naturaleza de las alteraciones visuales detectadas, ya que la única información concluyente sobre esto es la transmitida por los padres durante las consultas. Es por esto por lo que no se pudo concretar la concordancia de Atención Primaria y Oftalmología. Por lo tanto, al igual que en otros estudios, ${ }^{(8)}$ se considera necesaria una mejora de la relación y comunicación entre los diferentes servicios, que favorezca la accesibilidad e intercambio de información.

Uno de los problemas con los que se encuentran los programas de detección de trastornos visuales no realizados por oftalmólogos en la infancia, es el elevado número de falsos positivos, motivado por la falta de colaboración debido a la poca edad de los pacientes. Siendo en algunos estudios ${ }^{(9)}$ este porcentaje cercano al $75 \%$. No obstante, otros autores ${ }^{(2)}$ refieren que el grado de relación en España entre los defectos detectados en Atención Primaria y los confirmados por el servicio de Oftalmología de referencia varía entre un $93,6 \%$ y un $40 \%$.

Aunque alrededor del $40 \%$ de los participantes no se les realizó el test TNO, sólo existió la evidencia de que en el $9 \%$ del total de los casos esto ocurrió por la falta de colaboración en la realización del test; resultados similares, en torno al 9\%, a otros estudios ${ }^{(10,11)}$ sobre la aplicabilidad de test de visión estereoscópica.

En otros estudios ${ }^{(10)}$ sobre la detección de alteraciones visuales en Atención Primaria a través de test de visión estereoscópica (como es el TNO), si se pudo contrastar 
aquellos resultados anómalos derivados a Oftalmología, obteniéndose una confirmación de una alteración visual en el $73,7 \%$ de los casos, los cuales se pudieron beneficiar de un tratamiento y seguimiento precoz. En este estudio, de los siete casos con resultado anómalo en el test TNO, se debe remarcar uno de ellos en el que hasta realizar este test todas las demás pruebas diagnósticas iniciales eran normales; precisamente en virtud de la anomalía detectada en el test TNO y la repetición consecuente de las pruebas, se pudo determinar anomalías que conllevaron derivación al servicio de Oftalmología de referencia.

Se coincide con otras investigaciones ${ }^{(10)}$ en que los test de visión estereoscópica, como el test TNO, son aplicables a partir de los 3 años en las consultas de Atención Primaria y en que la consulta de Enfermería Pediátrica ofrece un ambiente idóneo para la colaboración del paciente en la ejecución de estas pruebas. Otros autores ${ }^{(12)}$ resaltan que la eficacia de estos test está relacionada con la edad, pues al acercarse a la edad de culminación del desarrollo visual, los resultados más específicos (en los que es necesario un mayor grado de agudeza binocular) mejoran considerablemente. Al igual que en otros estudios ${ }^{(9)}$ se coincide en la idea que implantar un sistema de despistaje de las alteraciones visuales llevado exclusivamente por oftalmólogos sería demasiado costoso. Del mismo modo, se considera que los centros de Atención Primaria, durante las revisiones infantiles de salud, se encuentran capacitados para llevar a cabo un importante primer cribado en la detección precoz de los trastornos visuales.

\section{CONCLUSIONES}

Los test visuales utilizados en las consultas de Enfermería de Atención Primaria constituyen una herramienta fundamental en la detección precoz de anomalías visuales.

En el Programa de Salud Infantil del Gobierno de Canarias, la agudeza visual por Optotipos es considerada la prueba visual fundamental en las revisiones infantiles en Atención Primaria. Por este motivo, representa la prueba más ejecutada en los 358 casos que conforman la población de estudio. El resto de las pruebas visuales, al no estar señaladas con la misma importancia, se han efectuado de manera dispar.

El test TNO es una prueba viable en Centros de Atención Primaria, aplicable desde temprana edad, detectándose casos anómalos desde los 4 años de edad. Esta prueba ha resultado determinante en alguno de los casos estudiados.

Las pruebas del test de Hirschberg y el Cover test, a pesar de ser utilizados en más del $45 \%$ de los casos, ofrecen el menor número de resultados anómalos. Por el contrario, el test TNO demuestra una mayor eficacia en orden a la determinación de anomalías en los 358 casos del estudio.

En la mayoría de las historias clínicas de salud se ha referido la observación directa de Estrabismo, previo a la realización de las pruebas visuales, determinándose sólo un porcentaje del $3 \%$. Por lo tanto, en orden a la detección precoz de la ambliopía se requiere la realización de pruebas diagnósticas visuales.

Con objeto de valorar la concordancia de datos entre Atención Primaria y Especializada, se considera necesaria una mejora de la relación y comunicación entre los diferentes servicios, que favorezca la accesibilidad e intercambio de información. 


\section{REFERENCIAS}

1.- García Aguado J. Grupo PrevInfad. Cribado de alteraciones visuales en la infancia. En Recomendaciones Prevlnfad/PAPPS [en línea]. Actualizado noviembre de 2013 [fecha de acceso 10 de enero de 2015]. URL disponible en: http://www.aepap.org/previnfad/Vision.htm

2.- Delgado Domínguez JJ. Grupo PrevInfad/PAPPS. Detección de trastornos visuales ( $1^{a}$ parte). Rev Pediatr Aten Primaria [en línea]. 2008 [fecha de acceso 10 de enero de 2015]. Vol.10 (287-315). URL disponible en: http://www.pap.es/files/1116-838-pdf/951.pdf

3.- Serrano Camacho JC, Gaviria Bravo ML. Estrabismo y ambliopía, conceptos básicos para el médico de Atención Primaria. Medunab [en línea]. 2011 [fecha de acceso 10 de enero de 2015]. Vol. 14 (2, 108-120). URL disponible en:http://revistas.unab.edu.co/index.php?journal=medunab\&page=article\&op=view\&path $\% 5 \mathrm{~B} \% 5 \mathrm{D}=1561 \&$ path\%5B $\% 5 \mathrm{D}=1470$

4.- Martín Martín R, Bilbao Sustacha JA, Collado Cucò A. Vision screening in Primary Care: how it is performed?. Rev Pediatr Aten Primaria [en línea]. 2013 [fecha de acceso 30 de enero de 2015]. Vol. 15 (59, 221-227). URL disponible en:http://scielo.isciii.es/scielo.php?script=sci_arttext\&pid=S1139-

76322013000400004\&lng=en\&nrm=iso\&tlng=en

5.- Puertas Bordallo D. Oftalmología para pediatras de Atención Primaria. AEPap ed. Curso de Actualización Pediatría [en línea]. 2009 [fecha de acceso 30 de enero de 2015]. Madrid: Exlibris Ediciones. P.167-85. URL disponible en: https://familiaysalud.es/sites/default/files/oftalmologia.pdf

6.- Van Doorn LA, Evans JW, Edgar DF, Fortuin M. Manufacturer changes lead to clinically important differences between two editions of the TNO stereotest. Ophthalmic and Physiological Optics. [en línea] 2015. [fecha de acceso 20 de febrero de 2015]. Vol. 34(2, 243-249). Doi:10.1111/opo.12101. URL disponible en:http://openaccess.city.ac.uk/4867/1/OPO\%20proof\%2016-12-

13\%20version\%20van\%20Doorn\%20et\%20al.pdf

7.- Martín Martín R, Bilbao Sustacha JA. Collado Cucò A. Vision screening in Primary Care: how it is performed?. Rev Pediatr Aten Primaria. [en línea] 2013 [fecha de acceso 3 de marzo de 2015]. Vol.15 (59, 221-227).URL disponible en: http://scielo.isciii.es/scielo.php?script=sci_arttext\&pid=S1139-

76322013000400004\&lng=en\&nrm=iso\&tlng=en

8.- Verrone PJ, Simi MR. Prevalencia de agudeza visual baja y trastornos oftalmológicos en niños de seis años de la ciudad de Santa Fe. Arch Argent Pediatr. [en línea] 2008 [fecha de acceso 3 de marzo de 2015]. Vol. 106 (4, 328333). URL disponible en: http://www2.sap.org.ar/docs/publicaciones/archivosarg/2008/v106n4a08.pdf

9.- Abreu Reyes JA, Iceta González I, Quintana Herrera C. Estudio de las anomalías oculares en la población infantil de la Comarca de Acentejo (Isla de Tenerife). Arch Soc Canar Oftal. [en línea] 2004 [fecha de acceso 3 de marzo de 2015]. Vol. 15 (17-20). URL disponible en:http://www.oftalmo.com/sco/revista15/15sco04.htm

10.- Barriuso Lapresa LM. Función visual (niños de 9 meses a 3 años). Rev Pediatr Aten Primaria. [en línea] 2007 [fecha de acceso 3 de marzo de 2015]; Vol. 9. Suplemento 2; P.121-128. URL disponible en: http://pap.es/files/1116-673pdf/702.pdf

11.- Salazar Cuba V, Romanelli Zuazo A. Detección de ambliopía en niños de 3 a 6 años en el "Hospital del Niño Dr.Ovidio Aliaga Uría". Rev Soc Bol Ped. [en línea] 
2006 [fecha de acceso 3 de marzo de 2015]. Vol. 45 (3, 148-152). URL disponible en: http://www.scielo.org.bo/pdf/rbp/v45n3/v45n3a02.pdf

12.- Arias Díaz A, Bernal Reyes N, Pérez Martinto PC. Medición de agudeza visual estereoscópica en una población infantil sana. Rev. Mex. Oftalmol. [en línea] 2013. [fecha de acceso 30 de enero de 2015]. Vol. 87 (4, 215-219). URL disponible en: http://apps.elsevier.es/watermark/ctl_servlet? f=10\&pident_articulo=90268741\&pident_us $\underline{\text { uario }=0 \& \text { pcontactid }=\& \text { pident } \_ \text {revista }=321 \& \text { ty }=64 \& \text { accion }=\text { L\&origen }=\text { zonadelectura } \& w e b}$ $=$ www.elsevier.es\&lan $=e s \&$ fichero $=321 \mathrm{v} 87 \mathrm{n} 04 \mathrm{a} 90268741 \mathrm{pdf001.pdf}$ 